\title{
Potential Energy Surface Reconstruction and Lifetime Determination of Molecular Double-Core-Hole States in the Hard X-Ray Regime
}

\author{
T. Marchenko, ${ }^{1,2, *}$ G. Goldsztejn, ${ }^{1,3}$ K. Jänkälä, ${ }^{4}$ O. Travnikova, ${ }^{1,2}$ L. Journel, ${ }^{1,2}$ R. Guillemin, ${ }^{1,2}$ N. Sisourat, ${ }^{1}$ D. Céolin, ${ }^{2}$ \\ M. Žitnik, ${ }^{5}$ M. Kavčič, ${ }^{5}$ K. Bučar, ${ }^{5}$ A. Mihelič,${ }^{5}$ B. Cunha de Miranda, ${ }^{1}$ I. Ismail,${ }^{1}$ A. F. Lago, ${ }^{6}$ F. Gel'mukhanov, ${ }^{7,8}$ \\ R. Püttner, ${ }^{9}$ M. N. Piancastelli, ${ }^{1,10}$ and M. Simon ${ }^{1,2}$ \\ ${ }^{1}$ Sorbonne Universités, UPMC Univ Paris 06, CNRS, UMR 7614, Laboratoire de Chimie Physique-Matière et Rayonnement, \\ F-75005 Paris, France \\ ${ }^{2}$ Synchrotron SOLEIL, l'Orme des Merisiers, Saint-Aubin, BP 48, F-91192 Gif-sur-Yvette Cedex, France \\ ${ }^{3}$ Max-Born-Institut, Max-Born-Straße 2A, 12489 Berlin, Germany \\ ${ }^{4}$ Nano and Molecular Systems Research Unit, University of Oulu, P.O. Box 3000, 90014 Oulu, Finland \\ ${ }^{5}$ Jožef Stefan Institute, SI-1001 Ljubljana, Slovenia \\ ${ }^{6}$ Centro de Ciências Naturais e Humanas, Universidade Federal do ABC (UFABC), 09210-580 Santo André, SP, Brazil \\ ${ }^{7}$ Theoretical Chemistry \& Biology, School of Biotechnology, Royal Institute of Technology, SE-106 91 Stockholm, Sweden \\ ${ }^{8}$ Institute of Nanotechnology, Spectroscopy and Quantum Chemistry, Siberian Federal University, 660041 Krasnoyarsk, Russia \\ ${ }^{9}$ Fachbereich Physik, Freie Universität Berlin, D-14195 Berlin, Germany \\ ${ }^{10}$ Department of Physics and Astronomy, Uppsala University, PO Box 516, SE-751 20 Uppsala, Sweden
}

(Received 19 June 2017; published 27 September 2017)

A combination of resonant inelastic x-ray scattering and resonant Auger spectroscopy provides complementary information on the dynamic response of resonantly excited molecules. This is exemplified for $\mathrm{CH}_{3} \mathrm{I}$, for which we reconstruct the potential energy surface of the dissociative I $3 d^{-2}$ double-core-hole state and determine its lifetime. The proposed method holds a strong potential for monitoring the hard $\mathrm{x}$-ray induced electron and nuclear dynamic response of core-excited molecules containing heavy elements, where $a b$ initio calculations of potential energy surfaces and lifetimes remain challenging.

DOI: 10.1103/PhysRevLett.119.133001

Hard x-ray radiation, with photon energy above $1 \mathrm{keV}$, can excite deep core shells of heavy atoms ( $\mathrm{Cl} 1 s, \mathrm{~S} 1 s, \mathrm{I} 2 p$, etc.) and may cause breakage of the chemical bonds and drastic changes to the electronic structure. The interplay between the induced nuclear and electron dynamics determines the response of a core-excited molecule. In the case of resonant excitation to a dissociative molecular state, the time evolution of the relaxation process is determined by the potential energy surface (PES) and the lifetime of the excited state. The so-called "core-hole clock" spectroscopy (CHCS) allows probing ultrafast dynamics, occurring in a resonantly coreexcited molecule within the core-hole lifetime, through control over the photon energy [1-4].

Resonant inelastic x-ray scattering (RIXS) and resonant Auger electron spectroscopy (RAS) are the two CHCS techniques relevant, respectively, to the measurements of $\mathrm{x}$-ray photons or Auger electrons emitted in the course of relaxation of core-excited molecular states. As the probability of radiative relaxation of core-excited atoms increases with atomic number, both RIXS and RAS become equally relevant in the hard X-ray regime [5-14]. However, the major difference between these techniques appears in the electronic final states reached upon relaxation. In the case of RIXS, the molecule remains neutral with an electron in the excited orbital and a single hole, whereas in the case of resonant spectator Auger decay, the molecule becomes singly charged with an electron in the excited orbital and a double hole.
Spectroscopy of double core-hole (DCH) states, created either with intense XFEL radiation through multiphoton absorption [15-17] or with high-energy photons provided by synchrotron radiation [18-23], has been a hot topic in recent years. The quantitative understanding of hard x-rayinduced processes in polyatomic molecules [24], including nuclear dynamics of DCH states in molecules, formed in the course of cascade relaxations, is of particular interest in relation to radiation-induced damage in organic tissue and coherent diffraction imaging $[25,26]$.

In this Letter, we demonstrate an original method to extract information on the electron and nuclear dynamic response of molecules in DCH states, created with hard x-ray radiation. In contrast to the soft $\mathrm{x}$-ray region, where nuclear dynamics occurs in a core-excited state, a short lifetime of a coreexcited state in the hard $\mathrm{x}$-ray regime allows a simple and, hence, robust way to reconstruct the PES that affects the evolution of a nuclear wave packet (WP) in the final state. Furthermore, we obtain an estimate of the I $3 d^{-2}$ DCH state lifetime, which determines the dynamics of the electronic structure evolution at the subsequent relaxation step. Our experimental method is validated by comparing the results obtained for the $\mathrm{CH}_{3} \mathrm{Cl}$ molecule with ab initio calculations of PES and the value of the $\mathrm{Cl} 2 p^{-2} \mathrm{DCH}$ lifetime estimated with other experimental methods. The advantage of our approach is especially significant in molecules, such as $\mathrm{CH}_{3} \mathrm{I}$, that contain heavy multielectron atoms and, thus, present a 
serious challenge for $a b$ initio description due to a large number of shells and the necessity to account for the electronic relaxation effects.

The RIXS measurements were performed at the ID26 beam line at the European Synchrotron Radiation Facility (ESRF). The experimental setup and the experimental settings are described in detail in Refs. [12,27]. The RIXS spectra corresponding to the $L_{3} M_{4,5}$ transition were acquired with a total instrumental resolution of $\approx 0.65 \mathrm{eV}$ determined from the width of the elastic peak, which includes the incident photon bandwidth and the spectrometer resolution.

The RAS measurements were performed at the end station HAXPES, based on a hemispherical electron analyzer installed on the GALAXIES beam line at the synchrotron SOLEIL $[28,29]$. The spectrometer is set parallel to the light polarization vector. The $L_{3} M_{4,5} M_{4,5}$ Auger spectra were recorded with the total resolution of $\approx 0.6 \mathrm{eV}$, which includes the photon bandwidth, the spectrometer resolution and the thermal Doppler broadening.

The RIXS and RAS measurements are presented in Fig. 1 in the form of 2D maps, obtained by recording the spectra while changing the incident photon energy with a step of $0.2 \mathrm{eV}$ around the I $L_{3}$ edge. Below the ionization threshold located around $4565 \mathrm{eV}$, both maps contain resonant lines dispersing with photon energy. The resonant excitation from the I $2 p_{3 / 2}$ shell to the lowest unoccupied molecular orbital (LUMO) $15 a_{1}$ occurs at $4559.2 \mathrm{eV}$ photon energy. Above ionization threshold the maps display the onset of nonresonant $L_{3} M_{4,5}$ fluorescence and $L_{3} M_{4,5} M_{4,5}$ normal Auger lines independent of the photon energy. The rich multiplet Auger structure is assigned in the Supplemental Material [30]. For a further analysis, we select in both maps a well-separated resonant line marked with a circle and trace its spectral shape as a function of photon energy around the $2 p_{3 / 2} \rightarrow 15 a_{1}$ resonant transition. The final state configuration for the selected lines is $3 d_{5 / 2}^{-1} \mathrm{LUMO}^{*}$ in the case of RIXS and $\left(3 d_{5 / 2}^{-2}\right)_{4} \mathrm{LUMO}^{*}$ in the case of RAS. Figure 1(c) shows the full width at half maximum (FWHM) of the selected RIXS and Auger lines as a function of the incident photon energy around the LUMO absorption resonance. The error bars are extracted from the fit of the experimental spectra with Voigt profiles using the SPANCF macro package [31,32].

The cross section for RIXS and RAS in the case of dissociative intermediate and final states is described by the generalized Kramers-Heisenberg formula [33]. In the Franck-Condon and Born-Oppenheimer approximations the dipole and Coulomb transition matrix elements are independent of the nuclear coordinates and the expression for the cross section for a given final state simplifies to

$$
\begin{aligned}
\sigma\left(\omega, \omega^{\prime}\right) \propto & \int_{0}^{\infty} d E_{f}\left|\sum_{c} \frac{\left\langle\chi_{f} \mid \chi_{c}\right\rangle\left\langle\chi_{c} \mid \chi_{o}\right\rangle}{\omega-\omega_{c o}-\Delta E_{c}+\imath \Gamma_{c}}\right|^{2} \\
& \times L\left(\omega-\omega^{\prime}-\omega_{f o}-\Delta E_{f}, \Gamma_{f}\right) .
\end{aligned}
$$

(a)

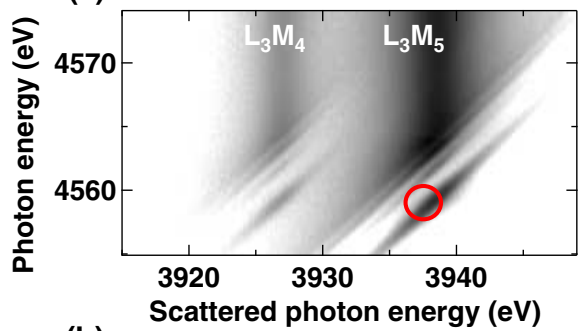

(b)

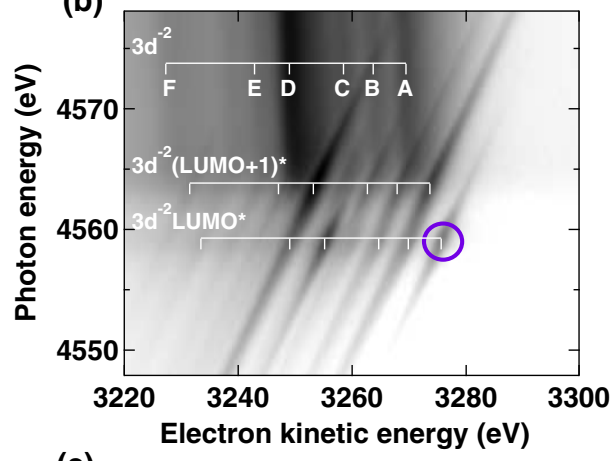

(c)

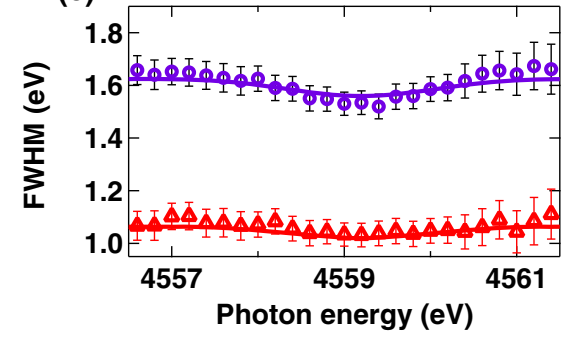

FIG. 1. (a) RIXS $L_{3} M_{4,5}$ and (b) Auger $L_{3} M_{4,5} M_{4,5}$ spectra recorded in $\mathrm{CH}_{3} \mathrm{I}$ near the I $L_{3}$ edge. A detailed assignment of the labeled Auger lines is presented in the Supplemental Material [30]. (c) FWHM of RIXS emission line (triangles) and Auger line (circles) selected in (a) and (b), respectively, as a function of photon energy around the LUMO resonance. Error bars are obtained from the fits of the experimental spectra. Solid lines show simulations based on Eqs. (2) and (3).

Here, the subscripts $i=f, c$, and $o$ stand, respectively, for the final, intermediate, and ground molecular states, $\omega$ is the incident photon energy, $\omega^{\prime}$ is the energy of the scattered photon or of the ejected Auger electron, $\omega_{j o} \equiv U_{j}\left(r_{0}\right)-\mathcal{E}_{o}$ is the energy of the $o \rightarrow j=c, f$ vertical transition, $\mathcal{E}_{o}$ is the total energy of the ground state (electronic + nuclear), $\Gamma_{c, f}$ are the lifetime broadenings of the intermediate and final states described by the dissociative potentials $U_{c, f}(r), \Delta E_{c, f}=E_{c, f}-\Delta U_{c, f}$ are the local nuclear kinetic energies at the point of the vertical transition $r=r_{0}$, $\Delta U_{c, f} \equiv U_{c, f}\left(r_{0}\right)-U_{c, f}(r \rightarrow \infty)$, and $E_{c, f}$ are the nuclear kinetic energies at $r \rightarrow \infty$. The numerator in Eq. (1) contains Franck-Condon (FC) overlaps between the nuclear wave functions $\chi_{i}$ and $L\left(\omega-\omega^{\prime}-\omega_{f o}-\Delta E_{f}, \Gamma_{f}\right)$ is the normalized Lorentzian function with the FWHM equal to $\Gamma_{f}$ that accounts for energy conservation throughout the whole absorption-decay process. 
In our case both the core-excited and final states are dissociative along the $\mathrm{C}-\mathrm{I}$ bond [12]. However, due to the large reduced mass and an extremely short lifetime of only 200 as of the I $2 p_{3 / 2}^{-1}$ core-excited state [34], we can neglect the propagation of the nuclear WP in the intermediate state and consider an instantaneous transfer [4] of the initial WP $\left|\chi_{o}\right\rangle$ to the final state at the equilibrium internuclear distance $r_{0}=2.139 \AA$. Consequently, the numerator in Eq. (1) describes a projection of the ground-state wave function localized near the equilibrium $r_{0}$ onto the continuum wave function in the final state $\left|\left\langle\chi_{f} \mid \chi_{o}\right\rangle\right|^{2}$. Furthermore, during fast scattering the nuclei have no time to change the local velocities as well as the local kinetic energies $\Delta E_{c} \approx \Delta E_{f} \equiv \epsilon$. Then the cross section reads

$$
\begin{aligned}
\sigma\left(\omega, \omega^{\prime}\right) \propto & \int_{-\Delta U_{f}}^{\infty} d \epsilon \exp \left(-\frac{\epsilon^{2}}{\Delta_{f}^{2}}\right) L\left(\omega-\omega_{c o}-\epsilon, \Gamma_{c}\right) \\
& \times L\left(\omega-\omega^{\prime}-\omega_{f o}-\epsilon, \Gamma_{f}\right),
\end{aligned}
$$

where $\Delta_{f}=\mathcal{F}_{f o} a_{o}$ is the width of the FC distribution connecting the ground and the final states, $\mathcal{F}_{f o}$ is the absolute value of the gradient of the PES of the dissociative final state at the equilibrium internuclear distance, $a_{o}=$ $\left[\left(\hbar / \mu \omega_{o}\right)\right]^{1 / 2}$ is the width of the nuclear WP in the ground state, $\mu$ is the reduced mass of the molecule with $(1 / \mu)=$ $\left(1 / m_{\mathrm{CH}_{3}}\right)+\left(1 / m_{I}\right)$, and $\omega_{o}$ is the vibrational frequency along the $\mathrm{C}-\mathrm{I}$ bond.

When the lifetime width of the final state is negligible $\Gamma_{f} \ll \Gamma_{c}, \Delta_{f}$, then $L\left(\omega-\omega^{\prime}-\omega_{f o}-\epsilon, \Gamma_{f}\right) \approx \delta\left(\omega-\omega^{\prime}-\omega_{f o}-\epsilon\right)$ and the cross section can be expressed analytically [35]:

$$
\begin{array}{r}
\sigma\left(\omega, \omega^{\prime}\right) \propto \exp \left(-\frac{\left(\omega-\omega^{\prime}-\omega_{f o}\right)^{2}}{\Delta_{f}^{2}}\right) \\
\times L\left(\omega^{\prime}-\omega_{c o}+\omega_{f o}, \Gamma_{c}\right) .
\end{array}
$$

This approximation is generally valid in the case of RIXS $[5,8,12]$. However, in the case of RAS, the lifetime broadening of the DCH final state may be non-negligible and the cross section must be evaluated using Eq. (2).

Convolution of the theoretical cross sections in Eqs. (2) and (3) with a Gaussian describing instrumental function allows for a direct comparison with the experimental results presented in Fig. 1(c). In our simulations we fixed the following parameters: $\omega_{c o}=4559.2 \mathrm{eV}, \omega_{f o}=620.9 \mathrm{eV}$ in the case of RIXS and $\omega_{f o}=1283.2 \mathrm{eV}$ in the case of Auger as follows from the experimental observations, $\Gamma_{c}\left(2 p_{3 / 2}^{-1}\right)=2.7 \mathrm{eV}$ [34], reduced mass of $\mathrm{CH}_{3} \mathrm{I}$ $\mu=13.4 \mathrm{amu}, \mathrm{C}-\mathrm{I}$ vibrational frequency $\omega_{o}=16 \mathrm{THz}$, which gives $a_{o}=0.07 \AA$. The unknown values for the lifetime broadening of the DCH final state in the case of Auger $\Gamma_{f}\left(3 d_{5 / 2}^{-2}\right)$ and the gradients $\mathcal{F}_{f o}\left(3 d_{5 / 2}^{-2}\right)$ and $\mathcal{F}_{f o}\left(3 d_{5 / 2}^{-1}\right)$ of the PES of the final states at the equilibrium internuclear distance were kept as free parameters and have been determined from the fit exhibiting an excellent agreement with the experimental data.
The line narrowing at the resonant absorption energy of $4559.2 \mathrm{eV}$ observed in both cases in Fig. 1(c) is due to the quenching of the vibrational broadening reflected by the FC distribution in the core-excited state and was previously observed in molecular RIXS and RAS spectra $[5,9,12]$. The overall larger FWHM for the Auger line as compared to the RIXS line reflects two important differences between the final states. Close to the resonance, where the linewidth is mainly determined by the lifetime broadening, the FWHM of the Auger line indicates a larger lifetime broadening of the final $3 d_{5 / 2}^{-2}$ DCH state with respect to the $3 d_{5 / 2}^{-1}$ single core-hole $(\mathrm{SCH})$ state. At the photon energies above or below the resonance, where the linewidth is strongly dependent on the FC dynamical broadening, the larger width of the Auger line indicates a larger width of the FC distribution $\Delta_{f}$ and, consequently, a more dissociative character of the $3 d_{5 / 2}^{-2} \mathrm{DCH}$ state as compared to the $3 d_{5 / 2}^{-1} \mathrm{SCH}$ state. In spite of the lack of ab initio calculations that could reproduce these differences, we can elucidate and quantify the observed effect directly from our measurements.

Our results provide us with two important findings. First, the extracted value $\Gamma_{f}\left(3 d_{5 / 2}^{-2}\right)=1.05 \pm 0.05 \mathrm{eV}$ shows that the lifetime $\tau \propto 1 / \Gamma$ of the DCH state is $0.6 \mathrm{fs}$, which is around 2 times shorter than the lifetime of the $\mathrm{SCH}$ state $\tau=1.2 \mathrm{fs}$, where $\Gamma_{f}\left(3 d_{5 / 2}^{-1}\right)=0.56 \pm 0.05 \mathrm{eV}$ [34]. The lifetimes of $1 s^{-2} \mathrm{DCH}$ and the corresponding $1 s^{-1} \mathrm{SCH}$ states were previously reported for $\mathrm{Ca}, \mathrm{V}, \mathrm{Cr}, \mathrm{Co}$, and $\mathrm{Zn}$ atoms [36], where the former were found to be 2.2 times shorter than the latter. In our recent work on $1 s^{-2} \mathrm{DCH}$ states in neon [23] and $2 p^{-2}$ DCH states in argon [37] we have reported the ratios of 2.9 and 2.8 , respectively. In order to validate our method, we have applied it to the previously measured RIXS and RAS in $\mathrm{CH}_{3} \mathrm{Cl}$ excited at the $\mathrm{Cl} K$ shell [9]. The extracted lifetime broadening of the $\mathrm{Cl} 2 p^{-2}$ state $\Gamma\left(2 p^{-2}\right)=0.25 \pm 0.02 \mathrm{eV}$, which is around $2.9 \pm 0.2$ times larger than $\Gamma\left(2 p^{-1}\right)=0.086 \mathrm{eV}$ [38], is consistent with the observations in argon [37]. The obtained value of the $\mathrm{Cl} 2 p^{-2}$ lifetime broadening explains also the discrepancy between the experimental data and simulations of the resonant Cl KLL Auger line using Eq. (3), where the lifetime of the final state was neglected [9]. As suggested in Ref. [23], the $\Gamma_{\mathrm{DCH}} / \Gamma_{\mathrm{SCH}}$ ratio must be dependent on the effective charge of the nucleus seen by electrons as well as on principal quantum number of the shells involved in the relaxation. For atoms with high atomic number and, consequently, large effective charge, the ratio is expected to converge to 2 . Our results are in line with this hypothesis and provide a test case for further theoretical modeling.

A second finding concerns the obtained absolute values of the PES gradients at the equilibrium $\mathrm{C}-\mathrm{I}$ interatomic distance: $\quad \mathcal{F}_{f o}\left(3 d_{5 / 2}^{-2}\right)=7.9 \pm 0.3 \mathrm{eV} / \AA, \quad \mathcal{F}_{f o}\left(3 d_{5 / 2}^{-1}\right)=$ $7.1 \pm 0.3 \mathrm{eV} / \AA$. A dissociative potential $U_{f}(r)$ can be described by an exponential shape [39]: 

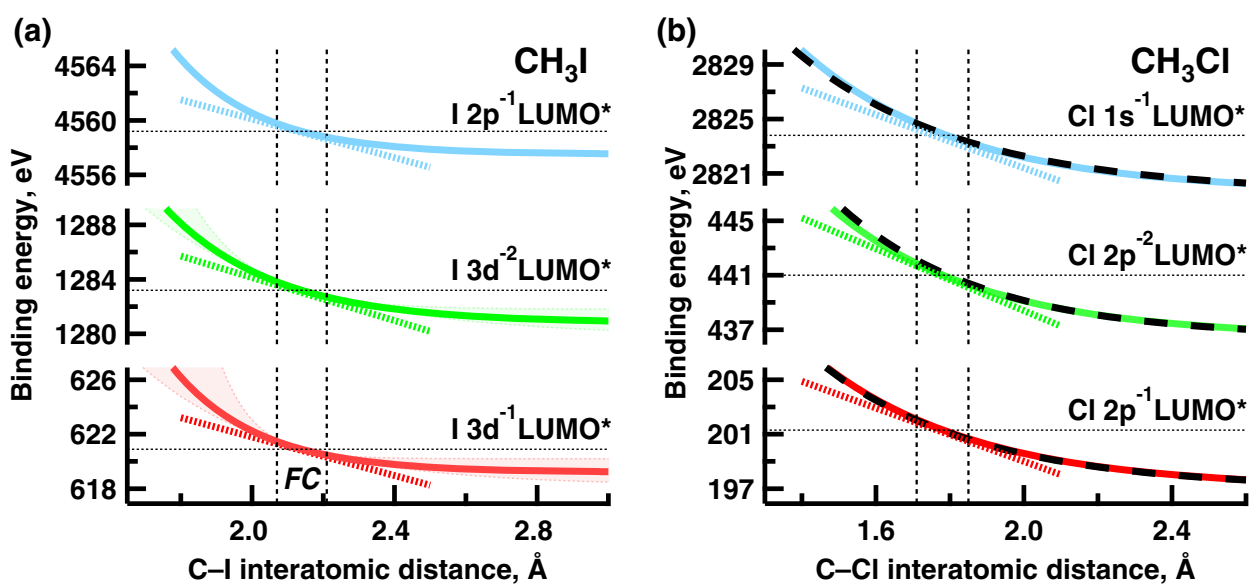

FIG. 2. PES reconstructed from the experimental data in (a) $\mathrm{CH}_{3} \mathrm{I}$ and (b) $\mathrm{CH}_{3} \mathrm{Cl}$ for the intermediate and final SCH and DCH states reached after RIXS or resonant Auger decay, respectively. The vertical lines mark the FC zone determined by the size $a_{o}$ of the groundstate vibrational wave function. The shaded area in a) corresponds to the uncertainty of $1 \mathrm{eV}$ for the asymptotic PES values calculated for atomic iodine. In $\mathrm{CH}_{3} \mathrm{Cl}$ the reconstructed PES of the final states (solid lines) are compared to the results obtained with ab initio calculations (dashed lines).

$U_{f}(r)=\Delta U_{f} \exp \left[\left(\frac{-\mathcal{F}_{f o}}{\Delta U_{f}}\right)\left(r-r_{0}\right)\right]+U_{f}(r \rightarrow \infty)$

Here, $U_{f}\left(r_{0}\right)$ is known from the experiment and the asymptotic PES value at large $\mathrm{C}-\mathrm{I}$ distances, where dissociation is completed, $U_{f}(r \rightarrow \infty)$, can be calculated from the transition energies in atomic iodine and the molecular dissociation energy in the ground state. Atomic transition energies were calculated using relativistic GRASP2K code [40] in the single configuration scheme. Note that in the case of RIXS, where both the intermediate and the final states are dissociative $\mathrm{SCH}$ states, their PES can often be considered as parallel to each other $[5,12]$. Therefore, we can assume the gradients of the I $2 p_{3 / 2}^{-1} \mathrm{LUMO}^{*}$ and $3 d_{5 / 2}^{-1} \mathrm{LUMO}^{*}$ states to be equal at $r_{0}$.

Figure 2(a) shows the simulated PES of the intermediate $2 p_{3 / 2}^{-1} \mathrm{LUMO}^{*}$, the final $3 d_{5 / 2}^{-1} \mathrm{LUMO}^{*} \mathrm{SCH}$, and the final $3 d_{5 / 2}^{-2} \mathrm{LUMO}^{*} \mathrm{DCH}$ states. This is an important outcome of our experimental analysis, since the existing theoretical tools for molecular PES calculations are so far not suitable for systems containing heavy multielectron elements. We confirm the reliability of our method using the case of $\mathrm{CH}_{3} \mathrm{Cl}$ excited at the $\mathrm{Cl} K$ shell [9] [Fig. 2(b)]. The extracted absolute values of the PES gradients at the equilibrium $\mathrm{C}-\mathrm{Cl}$ interatomic distance, $\mathcal{F}_{f o}\left(2 p^{-2}\right)=11.1 \pm 0.3 \mathrm{eV} / \AA$ and $\mathcal{F}_{\text {fo }}\left(2 p^{-1}\right)=10 \pm 0.3 \mathrm{eV} / \AA$, provide a perfect agreement between the PES simulated with our method using Eq. (4) and the results of $a b$ initio calculations using the MOLPRO package [41] at the CASSCF level, where we chose a Gaussian basis set aug-cc-pCVTZ $[42,43]$ to take into account core-core and core-valence correlation effects, and included relativistic corrections with the use of the DouglasKroll Hamiltonian $[44,45]$.

The obtained PES allow predicting the dissociation dynamics in the final states. We have adopted a method that uses an analytic representation of the WP based on Heller's semiclassical approach and has proven to provide an excellent agreement with the numerical solution of the time-dependent Schrödinger equation [39,46,47]. Figures 3(a) and 3(b) show the time evolution of the ordinary norm-conserving WP $|\chi(r, t)|^{2}$ in $\mathrm{CH}_{3}$ I along the simulated dissociative PES of the final $3 d_{5 / 2}^{-2} \mathrm{LUMO}^{*} \mathrm{DCH}$ and $3 d_{5 / 2}^{-1} \mathrm{LUMO}^{*} \mathrm{SCH}$ states, respectively. In both cases we can observe a narrowing of the WP width by 20\%-30\% from its initial value occurring around $t=10 \mathrm{fs}$ and a subsequent broadening during further evolution. The observed narrowing effect occurs in $\mathrm{CH}_{3} \mathrm{I}$ at times significantly exceeding the determined 0.6 fs lifetime of the DCH state when the population of this state is considerably depleted due to the Auger decay. However, it may play a role in molecules with light atomic constituents such as hydrogen halides undergoing fast dissociation [14].
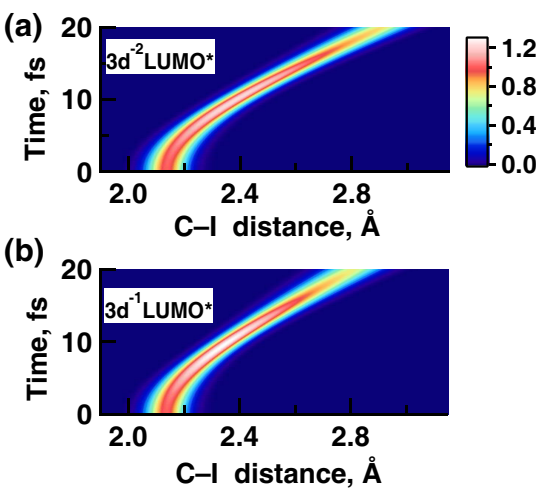

FIG. 3. Nuclear WP propagation along the simulated PES in (a) $3 d_{5 / 2}^{-2} \mathrm{LUMO}^{*} \mathrm{DCH}$ and (b) $3 d_{5 / 2}^{-1} \mathrm{LUMO}^{*} \mathrm{SCH}$ states. 
The predicted collapse of the WP width reflects the topology of the PES. A variation of PES gradient in the FC zone, determined by $d^{2} U_{f}\left(r_{o}\right) / d r^{2} \propto \mathcal{F}_{f o}^{2} / \Delta U_{f}$, sets the initial conditions for the continuum vibrational wave functions composing a coherent nuclear WP in such a way that the tail of the WP propagates with a higher phase velocity than its front. In the course of propagation, the tail components of the WP may catch up and eventually overtake the front, which results in a spatial narrowing and a subsequent broadening of the WP width.

In conclusion, we have demonstrated a novel experimental method to obtain information on the lifetime and PES of dissociative SCH and DCH states in molecules, core excited with hard $\mathrm{x}$-ray radiation. Our approach provides a unique opportunity to determine the time scales for evolution of electronic and molecular structure in molecules containing heavy multielectron atoms, where ab initio description so far remains challenging. The method can be directly applied to other quasidiatomic molecules where nuclear dynamics can be described with a dominant dissociative degree of freedom along the molecular axis. Generalization to polyatomic molecules with multiple dissociative degrees of freedom requires inclusion of multidimensional Franck-Condon factors in the adapted version of the Kramers-Heisenberg formula.

Experiments were performed on the GALAXIES beam line at SOLEIL Synchrotron, France (Proposal No. 20130944) and at the ID26 beam line at ESRF synchrotron. We are grateful to the SOLEIL staff for smoothly running the facility and to the ESRF staff for excellent assistance. T. M. acknowledges support of Agence Nationale de la Recherche, project ATTOMEMUCHO (ANR-16-CE30-0001). A. F. L. thanks Coordenação de Aperfeiçoamento de Pessoal de Nível Superior-Brazil for the support. F. G. acknowledges support within the Swedish Research Council (VR) and the State contract of the Russian Federation Ministry of Education and Science for Siberian Federal University for scientific research in 2017-2019 (Project 3.2662.2017).

\section{*tatiana.marchenko@upmc.fr}

[1] P. Morin and I. Nenner, Phys. Rev. Lett. 56, 1913 (1986).

[2] O. Björneholm, S. Sundin, S. Svensson, R. R. T. Marinho, A. Naves de Brito, F. Gel'mukhanov, and H. Ågren, Phys. Rev. Lett. 79, 3150 (1997).

[3] R. Feifel, F. Burmeister, P. Sałek, M. N. Piancastelli, M. Bässler, S. L. Sorensen, C. Miron, H. Wang, I. Hjelte, O. Björneholm et al., Phys. Rev. Lett. 85, 3133 (2000).

[4] F. Gel'mukhanov and H. Ågren, Phys. Rep. 312, 87 (1999).

[5] M. Simon, L. Journel, R. Guillemin, W. C. Stolte, I. Minkov, F. Gel'mukhanov, P. Sałek, H. Ågren, S. Carniato, R. Taïeb et al., Phys. Rev. A 73, 020706 (2006).

[6] L. Journel, L. El Khoury, T. Marin, R. Guillemin, S. Carniato, A. Avila, R. Delaunay, C. F. Hague, and M. Simon, Rev. Sci. Instrum. 80, 093105 (2009).
[7] M. Kavčič, M. Žitnik, K. Bučar, A. Mihelič, S. Carniato, L. Journel, R. Guillemin, and M. Simon, Phys. Rev. Lett. 105, 113004 (2010).

[8] R. Bohinc, M. Žitnik, K. Bućar, M. Kavčič, L. Journel, R. Guillemin, T. Marchenko, M. Simon, and W. Cao, J. Chem. Phys. 139, 134302 (2013).

[9] M. N. Piancastelli, G. Goldsztejn, T. Marchenko, R. Guillemin, R. K. Kushawaha, L. Journel, S. Carniato, J.-P. Rueff, D. Céolin, and M. Simon, J. Phys. B 47, 124031 (2014).

[10] G. Goldsztejn, T. Marchenko, D. Céolin, L. Journel, R. Guillemin, J.-P. Rueff, R. K. Kushawaha, R. Püttner, M. N. Piancastelli, and M. Simon, Phys. Chem. Chem. Phys. 18, 15133 (2016).

[11] O.Travnikova, T. Marchenko, G. Goldsztejn, K. Jänkälä, N. Sisourat, S. Carniato, R. Guillemin, L. Journel, D. Céolin, R. Püttner et al. Phys. Rev. Lett. 116, 213001 (2016).

[12] T. Marchenko, L. Journel, T. Marin, R. Guillemin, S. Carniato, M. Žitnik, K. Bučar, M. Kavčič, A. Mihelič, J. Hoszowska et al., J. Chem. Phys. 134, 144308 (2011).

[13] T. Marchenko, S. Carniato, L. Journel, R. Guillemin, E. Kawerk, M. Žitnik, M. Kavčič, K. Bučar, R. Bohinc, M. Petric et al., Phys. Rev. X 5, 031021 (2015).

[14] O.Travnikova, N. Sisourat, T. Marchenko, G. Goldsztejn, R. Guillemin, L. Journel, D. Céolin, I. Ismail, A. F. Lago, R. Püttner et al., Phys. Rev. Lett. 118, 213001 (2017).

[15] L. Young, E. P. Kanter, B. Krässig, Y. Li, A. M. March, S. T. Pratt, R. Santra, S. H. Southworth, N. Rohringer, L. F. DiMauro et al., Nature (London) 466, 56 (2010).

[16] N. Berrah, L. Fang, B. Murphy, T. Osipov, K. Ueda, E. Kukk, R. Feifel, P. Van der Meulen, P. Salen, H. T. Schmidt et al., Proc. Natl. Acad. Sci. U.S.A. 108, 16912 (2011).

[17] P. Salén, P. Van der Meulen, H. T. Schmidt, R. D. Thomas, M. Larsson, R. Feifel, M. N. Piancastelli, L. Fang, B. Murphy, T. Osipov et al., Phys. Rev. Lett. 108, 153003 (2012).

[18] J. H. D. Eland, M. Tashiro, P. Linusson, M. Ehara, K. Ueda, and R. Feifel, Phys. Rev. Lett. 105, 213005 (2010).

[19] P. Lablanquie, F. Penent, J. Palaudoux, L. Andric, P. Selles, S. Carniato, K. Bučar, M. Žitnik, M. Huttula, J. H. D. Eland et al., Phys. Rev. Lett. 106, 063003 (2011).

[20] M. Nakano, P. Selles, P. Lablanquie, Y. Hikosaka, F. Penent, E. Shigemasa, K. Ito, and S. Carniato, Phys. Rev. Lett. 111, 123001 (2013).

[21] S. Carniato, P. Selles, P. Lablanquie, J. Palaudoux, L. Andric, M. Nakano, Y. Hikosaka, K. Ito, T. Marchenko, O. Travnikova et al., Phys. Rev. A 94, 013416 (2016).

[22] R. Püttner, G. Goldsztejn, D. Céolin, J.-P. Rueff, T. Moreno, R. K. Kushawaha, T. Marchenko, R. Guillemin, L. Journel, D. W. Lindle et al., Phys. Rev. Lett. 114, 093001 (2015).

[23] G. Goldsztejn, T. Marchenko, R. Püttner, L. Journel, R. Guillemin, S. Carniato, P. Selles, O. Travnikova, D. Céolin, A. F. Lago et al., Phys. Rev. Lett. 117, 133001 (2016).

[24] A. Rudenko, L. Inhester, K. Hanasaki, X. Li, S. J. Robatjazi, B. Erk, R. Boll, K. Toyota, Y. Hao, O. Vendrell et al., Nature (London) 546, 129 (2017).

[25] M. M. Seibert, T. Ekeberg, F. R. N. C. Maia, M. Svenda, J. Andreasson, O. Jönsson, D. Odić, B. Iwan, A. Rocker, D. Westphal et al., Nature (London) 470, 78 (2011). 
[26] S. Boutet, L. Lomb, G. J. Williams, T. R. M. Barends, A. Aquila, R. B. Doak, U. Weierstall, D. P. DePonte, J. Steinbrener, R. L. Shoeman et al., Science 337, 362 (2012).

[27] M. Kavčič, M. Budnar, A. Muhleisen, F. Gasser, M. Žitnik, K. Bućar and R. Bohinc, Rev. Sci. Instrum. 83, 033113 (2012).

[28] D. Céolin, J. M. Ablett, D. Prieur, T. Moreno, J.-P. Rueff, T. Marchenko, L. Journel, R. Guillemin, B. Pilette, T. Marin et al., J. Electron Spectrosc. Relat. Phenom. 190, 188 (2013).

[29] J.-P. Rueff, J. M. Ablett, D. Céolin, D. Prieur, T. Moreno, V. Balédent, B. Lassalle, J. E. Rault, M. Simon, and A. Shukla, J. Synchrotron Radiat. 22, 175 (2015).

[30] See Supplemental Material at http://link.aps.org/ supplemental/10.1103/PhysRevLett.119.133001 for assignment of $L_{3} M_{4,5} M_{4,5}$ Auger lines in $\mathrm{CH}_{3} \mathrm{I}$ at the $\mathrm{I}_{3}$ edge.

[31] E.Kukk, G.Snell, J. D. Bozek, W.-T.Cheng, and N.Berrah, Phys. Rev. A 63, 062702 (2001).

[32] E. Kukk, K. Ueda, U. Hergenhahn, X.-J. Liu, G. Prümper, H. Yoshida, Y. Tamenori, C. Makochekanwa, T. Tanaka, M. Kitajima, and H. Tanaka, Phys. Rev. Lett. 95, 133001 (2005).

[33] T. Åberg and B. Crasemann, X-ray Resonant Anomalous Scattering (Elsevier, Amsterdam, 1994).

[34] J. L. Campbell and T. Papp, At. Data Nucl. Data Tables 77, 1 (2001).
[35] F. Gel'mukhanov and H. Ågren, Phys. Rev. A 54, 379 (1996).

[36] M. Polasik, K. Slabkowska, J. Rzadkiewicz, K. Koziol, J. Starosta, E. Wiatrowska-Koziol, J.-Cl. Dousse, and J. Hoszowska, Phys. Rev. Lett. 107, 073001 (2011).

[37] M. Žitnik, R. Püttner, G. Goldsztejn, K. Bučar, M. Kavčič, A.Mihelič, T. Marchenko, R. Guillemin, L. Journel, O. Travnikova, D. Céolin, M. N. Piancastelli, and M. Simon, Phys. Rev. A 93, 021401(R) (2016).

[38] M. Kivilompolo, A. Kivimäki, M. Jurvansuu, H. Aksela, S. Aksela, and R. F. Fink, J. Phys. B 33, L157 (2000).

[39] Z. W. Gortel, R. Teshima, and D. Menzel, Phys. Rev. A 60, 2159 (1999).

[40] P. Jönsson, X. He, C. Froese Fischer, and I. P. Grant, Comput. Phys. Commun. 177, 597 (2007).

[41] H.-J. Werner, P. J. Knowles, G. Knizia, F. R. Manby, and M. Schütz, Comput. Mol. Sci. 2, 242 (2012).

[42] T. H. Dunning, J. Chem. Phys. 90, 1007 (1989).

[43] D. E. Woon and T. H. Dunning, J. Chem. Phys. 98, 1358 (1993).

[44] M. Reiher and A. Wolf, J. Chem. Phys. 121, 2037 (2004); 121, 10945 (2004).

[45] A. Wolf, M. Reiher, and B. A. Hess, J. Chem. Phys. 117, 9215 (2002).

[46] E. J. Heller, J. Chem. Phys. 62, 1544 (1975).

[47] S.-Y. Lee and E. J. Heller, J. Chem. Phys. 71, 4777 (1979). 